Success in sight

\title{
The eyes have it! Ocular gene therapy trials for LCA look promising
}

JW Bainbridge and RR Ali

Gene Therapy (2008) 15, 1191-1192; doi:10.1038/gt.2008.117

The most severe forms of inherited blindness are collectively known as Leber congenital amaurosis (LCA) and are the first type of inherited blindness to be treated by gene therapy. Initial results from two separate clinical trials have been reported recently and are very encouraging for the field of ocular gene therapy.

LCA, originally described by Theodore Leber in 1869, is a group of recessively inherited severe infantileonset rod-cone dystrophies that is now known to be caused by defects in a variety of genes that encode proteins involved either in phototransduction, vitamin A cycling, photoreceptor development, ciliary transport function or phagocytosis. Defects in the gene, RPE65, which encodes a retinal isomerase responsible for regenerating visual pigment after exposure to light, cause impaired vision from birth and a progressive degeneration that leads to complete blindness in early adulthood. Currently there is no treatment. Although the retinal dystrophy caused by defects in RPE65 is severe, features of the disorder suggest that it may respond to gene-replacement therapy. A relatively well-preserved retinal structure, despite poor function, offers the possibility of detecting an improvement in vision within a few months of gene transfer. Proof of concept that gene therapy can result in a sustained improvement in retinal function was previously demonstrated in the Briard dog, a naturally occurring animal model of LCA caused by defects in RPE65.

In February 2007, the first clinical trial of gene therapy for LCA began in London at UCL Institute of Ophthalmology and Moorfields Eye Hospital. By the end of the year, two additional trials had started in the United States-one at Scheie's Center for Hereditary Retinal Degenerations, University of Pennsylvania and the University of Florida College of Medicine in Gainesville and an- other at the Children's Hospital of Philadelphia and the University of Pennsylvania. In April 2008 the initial results of two of these trials were published in the New England Journal of Medicine., ${ }^{1,2}$ Both groups report the first phase of their respective trials, which had a strong emphasis on safety and included only adult participants with advanced disease. The results demonstrate that the technique is feasible, appears to be safe in the short term and can lead to improvement in visual function within a period of months, even at a relatively late stage of the disease.

In both studies, a recombinant AAV2 vector was used to deliver a human RPE65 cDNA to the target retinal pigment epithelium cells. Although Maguire et al. ${ }^{2}$ used an AAV-2 vector with a constitutive promoter to drive transgene expression, Bainbridge et al. ${ }^{1}$ used elements of the endogenous RPE65 promoter. Subretinal vector injection, which causes a temporary retinal detachment, was achieved in all participants in the two trials without intraoperative complications and the detachments resolved spontaneously as anticipated. The subsequent development of a macular hole in one case was not clinically significant because the patient had very poor pre-existing vision; however, this complication would be relevant in a patient with better baseline function and illustrates the risks of surgery involving atrophic retina. There were no significant intraocular inflammatory responses to vector injection and no sustained reduction in visual function despite temporary retinal detachment. The long-term ocular and systemic safety of the procedure will be known only after further follow up.

Maguire et al. ${ }^{2}$ reported objective evidence of improved retinal function in all three patients in their study. Their study used the highly sensitive technique of pupillometry, which can detect responses to light of small numbers of functioning retinal cells by measuring the pupillary light reflex. Following subretinal vector injection, the pupillary response to light by the injected eyes was in each case stronger than that of the contralateral uninjected eye. Although neither group has detected an improvement in retinal function on electroretinography in any of their participants, this technique is relatively insensitive. In the context of retinal degeneration, an undetectable response on electroretinography is by no means inconsistent with useful visual function. The demonstration of improved retinal responses in these adult participants by pupillometry but not by electroretinography suggests the extent of the effect of gene therapy at this level of degeneration is relatively modest.

Both groups reported improvements in subjective measures of vision. Visual acuity measurements improved in the injected eyes of four out of the six participants who took part in the two trials. However, the measurement of acuity at very low levels of vision can be unreliable, and improvements were also observed in the uninjected eyes of two participants, so the significance of these findings is uncertain at present.

Bainbridge et al. ${ }^{1}$ detected a progressive improvement in retinal sensitivity in one patient using both microperimetry and dark-adapted perimetry. This effect was statistically significant and associated with a substantial improvement in visually guided mobility in dim light. This was demonstrated by an ability to negotiate a specially constructed simulation of a night-time street scene. It is believed that this particular patient benefited significantly because his disease was less far advanced at baseline than that of the other patients in the study. The possibility that the observed improvement in visual function is a consequence of a non-specific trophic effect of surgery cannot be completely discounted because subretinal delivery of an appropriate control vector would be ethically unacceptable; however, such a mechanism is considered highly unlikely given the extensive supporting data. The questions relating to the duration for which visual improvement is maintained in the human participants, and whether further retinal degeneration is slowed, can be addressed only after several years. 
It is hugely encouraging to see that gene-replacement therapy for RPE65 can be effective, even in adults who have severely advanced disease. Preclinical work suggests that it is likely to be most effective in affected individuals at an early age because younger individuals have less advanced retinal degeneration. Now that the first phase of the clinical trials have demonstrated the feasibility and safety of subretinal vector delivery in adults, the inclusion of younger individuals with better baseline visual function is justified. This next phase of the trials will determine whether younger individuals respond with consistent improvements in visual function and may help define a window of opportunity for the most favourable timing of intervention. The effect of gene replacement therapy on visual acuity might also be influenced by pre-existing ambylopia resulting from stimulus deprivation during early childhood in affected individuals and it seems likely that the optimal timing of intervention will be during this critically sensitive period of visual development.

Although the protocols of the two trials share common features including the use of recombinant AAV vectors, important differences will yield complementary data that are expected to inform on the optimal vector design and delivery. The comparison between the effect of a constitutive promoter versus a tissue-specific promoter on safety and efficacy will be of particular interest. Already, these trials have established proof of principle of gene therapy for inherited retinal disease and they will pave the way for the development of gene therapy approaches for a broad range of eye disorders.

\section{Acknowledgements}

The authors are supported by the National Institute of Health Research Biomedical Research Centre for Ophthalmology at Moorfields Eye Hospital and UCL Institute of Ophthalmology.

Professor RR Ali is at the Department of Molecular Therapy, Institute of Ophthalmology, University College London, Bath Street, London EC1V 9EL, UK.

E-mail:r.ali@ucl.ac.uk

1 Bainbridge JW, Smith AJ, Barker SS Robbie S, Henderson R, Balaggan K et al. Effect of gene therapy on visual function in leber's congenital amaurosis. $N$ Engl J Med 2008; 358: 2231-2239.

2 Maguire AM, Simonelli F, Pierce EA, Pugh Jr EN, Mingozzi F, Bennicelli J et al. Safety and efficacy of gene transfer for leber's congenital amaurosis. $N$ Engl J Med 2008; 358: 2240-2248. 